\title{
Société Française d'Allergie
}

\section{Séance du 18 Juin 1957}

Allergie hormonale

M. Pasteur Vallery-Radot. Deux questions se posent:

1) Peut-on se sensibiliser aux hormones, introduites par voie parentérale dans Torganisme? - 2)

Peut-on se sensibiliser à ses propres hormones?

A la premiere question répondent de nombreuses observations de sensibilisation à la suite d'injections d'hormones ovariennes, d'inhalation d'extrait hypophysaire ou d'injections d'A.C.T.H. II est aussi des allergies certaines à $\Gamma$ insuline. Dans le cas d'allergie à $\Gamma$ A.G.T.H. et d'allergie à $\Gamma$ insuline, $\Gamma$ auteur insiste sur le fait qu'il faut toujours se demander s'il s'agit d'une sensibilisation à Thormone elle-même ou aux protéínes de Гanimal dont elle est extraite. A la seconde question (autoallergie hormonale), Гauteur répond par les observations indiscutables d'auto-allergie aux hormones génitales de la femme. II indique comment, en pratique, reconnaître une allergie à ces hormones. Les autres autoallergies sont sujettes à critiques.

Allergie à ГA.C.T.H.

M. M. J. Charpin et A. J Gafiropoulo ont observe onze cas d'accidents allergiques dus à ГA.C.T.H. II s'est agi en regie générale d'urticaires, assorties ou non d'ædème de Quincke, et, trois fois, de crises d'asthme. Le plus souvent, une ou plusieurs injections antérieures avaient «prepare» le malade. Toutefoís, deux sujets ont réagi dès la premiere injection d'A.C.T.H. La nature des véhicules retard ne joue aucun role dans ces accidents. Les tests cutanés se sont toujours révélés négatifs aux difïerents solvants.

La provenance animale de Thormone n'est pas en cause non plus. Les malades ont été testés à la fois avec un produit d'origine porcine et un produit d'origine ovine: ces tests ont été également positifs. Parallèlement, des tests ont été pratiques avec les viandes de pore, de mouton et de bæuf; ils ont toujours été négatifs. Les tests ont été régulière-ment positifs à une hormone somatotrope d'origine bovine. Ces malades paraissent sensibilisés à une protéine hypophysaire se rencontrant surtout dans les différents extraits antéhypophysaires.

La frequence de ces accidents contre-indique peut-être chez les malades allergiques, Гusage périodique de ГA.C.T.H. au cours des traitements cortísoniques pro-longés.

M. Halpern. - L'introduction d'A.C.T.H. et son extension permettent de prévoir à priori qu'on allait créer des sensibilisations à cette hormone polypeptédique. Ce qui augmentait la chance de la sensibilisation, c'était la preparation des solutions dites «retard» par la precipitation de complexe forme avec le sel de zinc. La sensibilisation est due certainement à Thormone et non pas aux substances additionnelles; il a observe en effet des reactions allergiques violentes lors de Гinjection intraveineuse d'A.C.T.H. qui ne contient pas de substance «retard».

Avec une méthode nouvelle basée sur Tagglutination des érythrocytes copulés avec $\Gamma$ antigène à Гaide d'un pont benzidine, il a pu déceler, chez des malades allergiques à ГA.C.T.H., les anticorps spécifiques anti-A.C.T.H.

Proceedings - Gesellschaftsberichte - Sociétés 
343

Confrontation des résultats obtenus dans la detection des allergies médica-menteuses cutanées par les tests épicutanés et diverses techniques biologi-

ques nouvelles

M. M. E. Sidi et Reinberg ont observe, d'une part 57 sujets dont Thistoire clinique et la positivité des tests épicutanés permettaient d'affirmer les sensibilités médicamen-teuses et, d'autre part, 50 sujets témoins indemnes.

Sur 20 sensíbilisations détectées, chez 11 malades, la reaction néphélométrique était positive dans 6 cas.

En ajoutant au sang total une certaine quantité d'allergène médicamenteux, les auteurs ont observe, dans 10 cas sur 11, des modifications des globulines par électro-phorèse sur papier. Ils n'ont pas constate de reactions cytotoxiques, mais ils ont vu des phénomènes de crise suivie de recuperation des polynucléaires.

Dans un cas sur 7 malades sensibles au chloramphénicol, ils ont observe des phénomènes d'agglutination des hématies et des leucocytes.

Les auteurs pensent que, du point de vue pratique, le test épicutané reste la technique la plus sure et la plus sensible pour détecter Гorigine médicamenteuse d'une dermite allergique tissulaire. Etude endobronchique sur la dyspnée asthmatiforme chez l'animal et l'asthme chez l'homme M. M. Halpern et Dubois de Montreynaud présentent un film qui montre Гintérêt de la cinématographie et de la photographie endobronchique pour étudier la dyspnée asthmatiforme chez $\Gamma$ animal et l'asthme chez l'homme.

Chez Tanimal, $\Gamma$ excitation faradique du vague produit un spasme bronchique très net, plus prolongé après injection de prostigmine, surtout si celle-ci est suivie d'une injection d'acétylcholine.

Avec des aerosols d'histamine, conduits dans les bronches par un catheter, on obtient le plus souvent une importante hypersécrétion et une sténose des grosses bronches par spasme et cedème, disparaissant après aerosols de sympathicomimétiques. Si la sténose bronchique est plus lointaine, elle n'est pas visible au bronchoscope, mais elle se traduit par une dyspnée importante de $\Gamma$ animal.

Chez l'homme, on constate Гexistence d'un spasme bronchique à predominance expiratoire avec hypersécrétion et, dans les asthmes allergiques en particulier, la presence d'un cedème qui régresse après administration locale de sympathicomimétiques.

Etat actuel de la preparation des extraits allergéniques à l'Institut Pasteur

M. L. Guibert et Mme Combes. - L'Institut Pasteur prepare depuis peu des extraits allergéniques sees pour cuti-réactíons et des dilutions d'extraits pour intradermo-réactions et traitements de désensibilisation. Les cuti-réactions peuvent être également effectuées avec quelques gouttes des solutions les plus concentrées, utilisées pour les intradermo-réactions et les traitements, déposées sur le trait de scarification.

L'Institut Pasteur délivre actuellement: - pour cuti-réaction, un melange de pollens de graminées, des extraits sees de poussière de maison, de poils de chat, chien et lapin; de squames de cheval, de plumes. - pour intradermo-réactions et traitement de désensibilisation, des dilutions d'extrait de poussière de maison, de pollens de graminées, de moisisures et de champignons saprophytes: Aspergillus, Pénicillium, Cla-dosporium, Alternaria, Candida, Mucor. - Des extraits allergéniques (pour cuti- et intradermo-réaction) d'aliments divers (farine, æuf, lait etc.) sont en cours de preparation. 
Proceedings - Gesellschaftsberichte - Sociétés

Chélatiõn allergéniqne

M. Le Coulant et Mile Lopes mettent en evidence une utilisation possible, pratique, du blocage de Tallergénicité des derives du groupe du Chrome. Avec le calcitétracémate disodique et l'aminotriacétate de sodium, le blocage de Гallergénicité par Chelation est efficace. Ce blocage est relativement stable; il est spécifique des métaux lourds. Les auteurs ont trouvé que d'autres substances peuvent aussi, à des degrés variables, blo-quer Гallergénicité des derives du chrome: acide thiomalique, sulfites, thiosulfates, etc. Mais, ils préconisent surtout, en usage externe, des substances entrant normalement dans la constitution de Torganisme, comme la Cystéine (acide aminé de la peau et des phanères), dont ils expliquent le mode d'action. Bien des accidents d'irritation cutanée, de necrose et surtout d'allergie, dus en particulier aux derives du chrome, pourraient ainsi être évités sans risquer de provoquer une allergie nouvelle.

Examen de l'asthmatique en période de crise par bronchoscopie et spirographie

M. J. M. Dubois de Montreynaud recherche comment appliquer, à Гexamen courant des asthmatiques, des méthodes restées jusqu'ici dans le domaine scientifique.

La bronchoscopie peut être utíle chez l'asthmatique au cours d'un état de mal que $\Gamma$ on veut soulager ou pour écarter d'autres diagnostics différentiels. Elle permet de constater le spasme, de Thypersécrétion, de Гædème, une congestion de la muqueuse, signes différemment associés, et de les interpreter.

La spirographie permet d'étudier les tests les plus utiles: air courant, capacité vitale, reserves inspiratoire et expiratoire, volume respiratoire maximum seconde, etc. On peut déduire de ces tests «utílisables en routine» des notions très utiles concernant le type d'asthme, avec ou sans emphysème, les indications thérapeutiques, et $\Gamma$ action du traitement, le pronostic à porter.

L'étude fortuite comparée des aspects endobronchiques et des courbes spiro-graphiques, chez les mêmes malades, permet d'interpréter certains «accidents de courbe» et de porter ainsi un pronostic fâcheux ou favorable.

Pierre Blamoutier.

British Society for Immunology

The spring meeting of the British Society for Immunology was held in the Meyerstein Theatre of the Westminster Medical School, London, on May 10th and 11th, 1957. The program was:

/. G. Morris (University College, N. Wales): Experimentally induced haemolytic disease in young mice.

R. J. W. Rees (National Institute for Medical Research): Attempts to induce immune tolerance to M. tuberculosis in mice.

G. Belyavin (University College Hospital): The study of influenza virus antibodies by the direct flocculation reaction.

A. A. Miles (Lister Institute): The analysis of non-specific immunity.

G. Biozzi (Hospital Broussais, Paris): The phagocytic function of the reticulo-endothelial system and resistance to infections.

C. H. Lack (Royal National Orthopaedic Hospital): Some factors in susceptibility and resistance to tuberculosis.

E. Suter (University of Florida, U.S.A.): The effect of phagocytosis on respiration of microorganisms. 